\title{
Utility of Red Cell Distribution Width (RDW) In Diagnosis and Prognosis of Early-Onset Neonatal Sepsis in Term Neonates
}

\author{
Sandeep Golhar ${ }^{1}$, Abhishek Madhura ${ }^{2}$, Urmila Chauhan ${ }^{3 *}$, Abinash Nayak ${ }^{4}$ \\ ${ }^{1}$ Associate Professor, Department of Pediatrics, GMCH, Nagpur, India. \\ ${ }^{2}$ Assistant Professor, Department of Pediatrics, GMCH, Nagpur, India. \\ ${ }^{3}$ Associate Professor, Department of Pediatrics, AIIMS, Nagpur, India. \\ ${ }^{4}$ Ex-Junior Resident, Department of Pediatrics, GMCH, Nagpur, India.
}

Received 16 February 2021; Revised 09 May 2021; Accepted 19 June 2021; Published 01 September 2021

\begin{abstract}
Objective: To assess the increased Red Cell Distribution Width (RDW) in diagnosis and prognosis of early-onset neonatal sepsis in term neonates. Methods: In a prospective, observational study, we enrolled term neonates $(\geq 37$ weeks of gestation) clinically suspected for Early-Onset Neonatal Sepsis (EONS) (within 7 days of birth). A cut-off of $18 \%$ and above was taken to consider RDW as abnormal or increased. The primary outcome was to assess the relation of increased RDW with in-hospital mortality. The secondary outcome was to determine the diagnostic yield of increased RDW in culture-proven sepsis. Results: In 166 neonates, $60 \%$ were males. Increased RDW was seen in $42.42 \%$ of neonates and $15.75 \%$ of neonates had positive blood culture. Compared to normal RDW, in-hospital mortality was significantly higher in neonates with increased mortality $(27.14 \%$ vs. $10.52 \%$, respectively; $\mathrm{p}=0.006)$. Also, abnormal RDW was seen in $46.15 \%$ of neonates with positive blood culture compared to $35.25 \%$ of neonates with negative blood culture ( $\mathrm{p}<0.0001$ ). Thus, elevated RDW had a sensitivity of $44.4 \%$ and specificity of $57.97 \%$ in the diagnosis of EONS. Conclusion: Increased RDW can be a diagnostic as well as a prognostic marker in neonates with EONS. Such observation indicates it may serve as a simple and easily available marker for EONS in resource-limited settings. However, these findings need to be confirmed in a larger sample.
\end{abstract}

Keywords: Red Cell Distribution Width; Early-Onset Neonatal Sepsis; Mortality; Blood Culture; NICU.

\section{Introduction}

The neonatal period carries the highest risk of mortality. World Health Organization estimates for 2019 indicate that nearly 2.4 million neonates died worldwide [1]. Though the neonatal mortality in India decreased from 52 per 1000 live births in 1990 to 22 per 100 live birth in the year 2019, it remains substantially higher [2]. India alone contributes a quarter of neonatal deaths from South Asia [3]. Neonates are uniquely prone to invasive infective diseases because of their immature innate immunity [4]. Prematurity, neonatal infections, perinatal asphyxia, and congenital malformations are the major causes of neonatal mortality [5, 6]. Neonatal sepsis can be life-threatening. Nearly $15 \%$ of neonatal deaths are attributable to sepsis alone [7]. In India, the fatality rate due to neonatal sepsis ranges from 25 to $65 \%$ [8].

* Corresponding author: drurmilachauhan@gmail.com, drurmilachauhan@aiimsnagpur.edu.in

dol http://dx.doi.org/10.28991/SciMedJ-2021-0303-7

$>$ This is an open access article under the CC-BY license (https://creativecommons.org/licenses/by/4.0/).

(C) Authors retain all copyrights. 
Diagnosis of sepsis is challenging in neonates. Blood culture is a gold standard but is a time-consuming diagnostic method and its yield is affected by various factors [9]. Early prediction of neonatal sepsis using surrogate markers is necessary. Among various biomarkers for diagnosis of neonatal sepsis, C-reactive protein (CRP), procalcitonin (PCT), and interleukin 6 (IL-6) are considered most promising for diagnosing neonatal infections [10]. In sepsis pathophysiology, the inflammatory response is a vital part. Inflammatory response associated with sepsis suppresses the maturation process of RBC and increases their half-lives with a resultant rise in RDW levels [11, 12]. In recent studies, red cell distribution width (RDW) was identified as an important biomarker of disease severity in critically ill patients and was an independent predictor of mortality due to sepsis [11-14]. However, there is a relative lack of studies from India assessing RDW as a biomarker of diagnosing neonatal sepsis and predicting mortality. Hence, we performed this study to determine the utility of RDW in the diagnosis of early-onset neonatal sepsis (EONS) and assessed its association with neonatal mortality.

\section{Materials and Methods}

\subsection{Study Design and Population}

This study was a prospective, observational study, conducted at a tertiary care center from central India over a period of 2 years between October 2017 and October 2019. Term neonates ( $\geq 37$ weeks of gestation) clinically suspected for early-onset sepsis (defined as sepsis onset within day 7 of life) were included. All preterm neonates, birth asphyxia, meconium aspiration syndrome, $\mathrm{ABO} / \mathrm{Rh}$ incompatibility, very low birth weight, multiple pregnancy, and neonates with any congenital malformations were excluded. The study was approved by the Institutional ethics committee. Voluntarily given written informed consent was taken from either or both parents and/or caretakers of the neonates enrolled in the study.

\subsection{Definitions of Sepsis}

Neonatal sepsis was defined as per the criteria from the National Neonatal Forum of India as follows. Probable (clinical) sepsis was considered when infants had clinical picture suggestive of septicemia if there is the presence of any one of the following criteria: Existence of predisposing factors such as maternal fever or foul-smelling liquor or prolonged rupture of membranes $(>24 \mathrm{hrs}$.) or gastric polymorphs ( $>5$ per HPF); positive sepsis screen indicated by the presence of two out of four parameters namely, total leucocyte count (TLC) $<5000$ cells $/ \mathrm{cmm}$, band to total polymorphonuclear cell ratio of $>0.2$, absolute neutrophil count $<1800 / \mathrm{cmm}, \mathrm{CRP}>1 \mathrm{mg} / \mathrm{dl}$ and micro ESR $>10$ mmfirst hour and radiological evidence of pneumonia. Culture positive sepsis was diagnosed in infants with features suggestive of septicemia, pneumonia, or meningitis, if there is the presence of either isolation of pathogens from blood or CSF or urine or pathological evidence of sepsis on autopsy. EONS was considered to be present when the newborn presented with clinical features of sepsis at or before 72 hours of life.

\subsection{Clinical Examination}

On arrival, each neonate admitted to the neonatal intensive care unit (NICU) of our tertiary care hospital. General examination including anthropometric measures, vitals, skin examination was carried out in a well-illuminated room. Neonates with a birth weight of $<2500$ to 1500 gms, $<1500$ to 1000 gms and <1000 gms were considered as low-birthweight (LBW), very LBW (VLBW) and extremely LBW (ELBW) neonates. The presence of apnea, respiratory distress and hypothermia was noted. Apgar score was calculated using an extended Apgar score form.

\subsection{Laboratory Assessments}

Complete blood count (CBC), peripheral smear, CRP, random blood glucose, serum creatinine, blood culture, CSF studies, and x-ray were performed. From the CBC, we determined hemoglobin, total leucocyte count (TLC), differential count, platelet count and red cell distribution width (RDW). In full-term neonates, RDW range from $13 \sim 18 \%$ [15]. Furthermore, some studies found mean values $15.65 \pm 1.18$ and $16.7 \pm 1.6$ [16, 17]. Thus, to define increased RDW, we had taken a cut-off value of $\geq 18 \%$. C- reactive protein (CRP) was measured by slide agglutination test. Blood glucose estimation was done with point of care (POC) reagent strips.

Blood cultures were performed in all neonates and were observed at least for 72 hours. The presence or absence of visible growth was noted every 24 hours. The incubation period was seven days to rule out late-growing bacteria. Clinical isolates were identified by colony morphology, microscopy, and routine biochemical reaction. CSF examination for sugar, proteins and microscopy, gram staining, and culture sensitivity. Simultaneously, a blood sugar sample was collected to compare with CSF sugar.

\subsection{Outcome Measure}

The primary outcome was the utility of RDW in the prognosis of in-hospital death. The secondary outcome was to determine the diagnostic yield of increased RDW in culture-proven sepsis. 


\subsection{Data Analysis}

Based on the assumptions of estimated incidence of EONS 30\% [18] with an absolute precision of 7\% and desired confidence level of $95 \%$, a sample size of 165 was calculated. Data from the case record forms was entered into the Microsoft excel sheets were evaluated using SPSS software version 15.Categorical data were presented as frequency and percentages. Statistical significance in categorical variables was assessed using Chi-square or Fisher's exact test. Continuous data were presented as mean and standard deviation. The student's t-test was applied for continuous variables. P-value $<0.05$ considered as significant.

\section{Results}

In this study, we enrolled 165 neonates (age <28 days). Figure 1 describes the study methodology with distribution of number of neonates in various parameters.

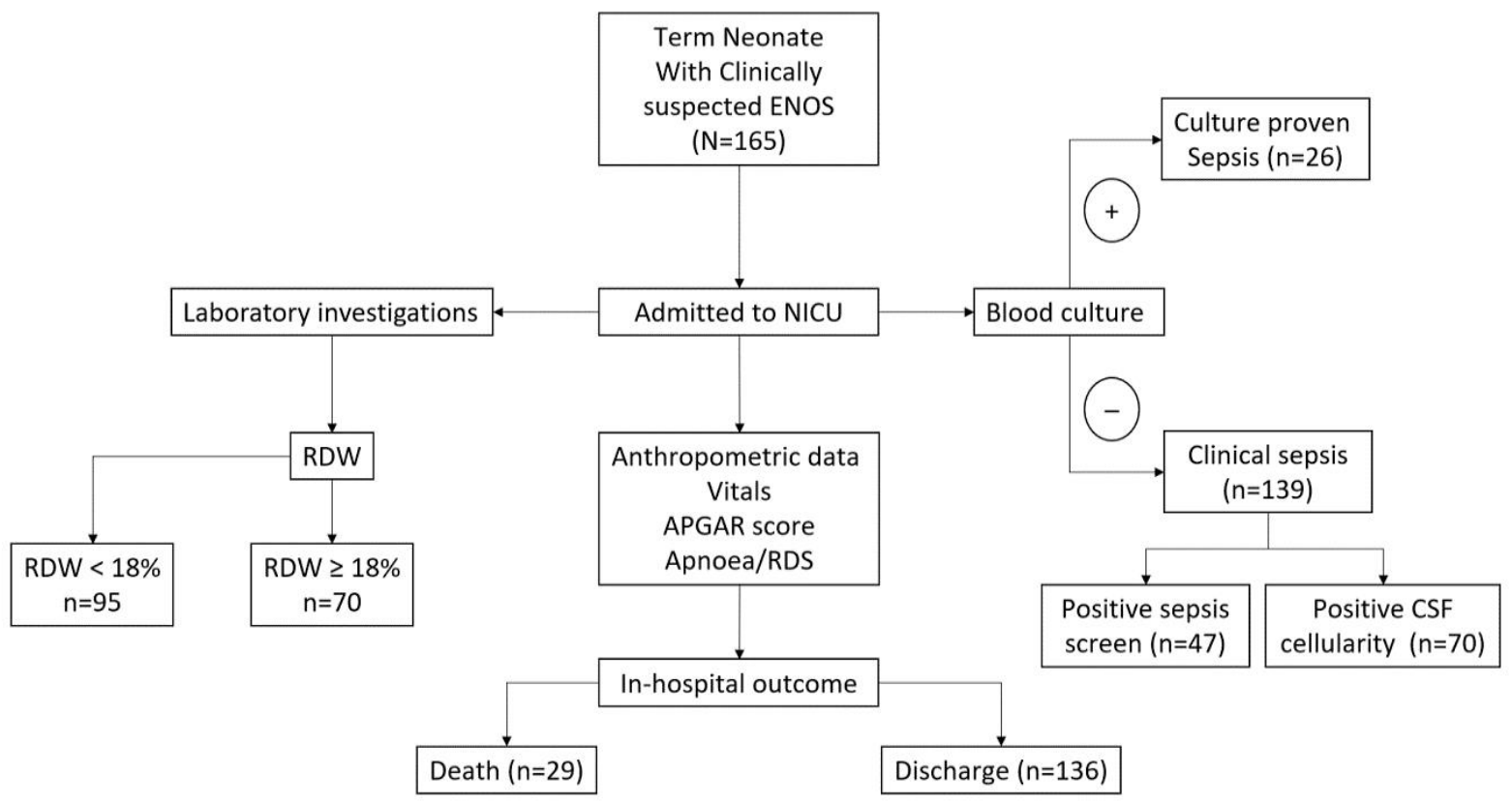

Figure 1. Study methodology with neonate flow in study

Table 1 describes the baseline characteristics of the enrolled neonates. Of them, $60 \%$ were of male sex with male to female ratio being 1.5:1. At birth, $47.27 \%$ were delivered in our hospital $21.21 \%$ were home delivered and $53.72 \%$ were outborn. Nearly half of the neonates had birth weight $<2 \mathrm{~kg}(50.91 \%)$ whereas nearly one-fourth had weight between 2 to $2.5 \mathrm{~kg}(24.24 \%)$. On arrival, the most common signs and symptoms included shock (82.42\%), and refusal to feed $(81.21 \%)$ followed by hypothermia $(64.24 \%)$, lethargy $(55.76 \%)$ and seizure $(53.94 \%)$, apnea (33.94\%), cyanosis $(32.73 \%)$. Nearly $30 \%$ were in distress. The most common maternal risk factor for early-onset neonatal sepsis observed was prolonged rupture of membranes $(52.12 \%)$ followed by foul smelling liquor $(41.82 \%)$, maternal fever $(41.82 \%)$ and unclean per vaginal examination $(12.12 \%)$. In hematological parameters, leukocytopenia was seen in $40.61 \%$ of neonates whereas $22.42 \%$ had absolute neutrophil counts (ANC) $<1500$ and $40 \%$ had thrombocytopenia. Increased RDW ( $\geq 18 \%$ ) was seen in $42.42 \%$ of neonates whereas $52.12 \%$ had elevated CRP.

Table 1. Baseline characteristics

\begin{tabular}{lc}
\hline Characteristics & Observations \\
\hline Gender & \\
\hline Male & $99(60.00)$ \\
Female & $66(40.00)$ \\
\hline Place of birth & \\
\hline Inborn & $78(47.27)$ \\
Out born & $52(53.72)$ \\
Home & $35(21.21)$ \\
\hline Mode of Delivery & \\
Vaginal & $95(57.58)$ \\
Cesarean Section & $70(42.42)$ \\
\hline
\end{tabular}




\begin{tabular}{lc}
\hline Birth weight & \\
\hline$>3 \mathrm{~kg}$ & $22(13.33)$ \\
$2.5-3 \mathrm{~kg}$ & $19(11.52)$ \\
$2.0-2.5$ & $40(24.24)$ \\
$<2 \mathrm{~kg}$ & $84(50.91)$ \\
\hline Signs and Symptoms & $136(82.42)$ \\
\hline Shock & $134(81.21)$ \\
Refusal to feed & $106(64.24)$ \\
Hypothermia & $92(55.76)$ \\
Lethargy & $89(53.94)$ \\
Seizure & $56(33.94)$ \\
Apnea & $54(32.73)$ \\
Cyanosis & $50(30.30)$ \\
Hypoglycemia & $49(29.70)$ \\
Distress & $39(23.64)$ \\
Abdominal distension & $85(52.12)$ \\
\hline Maternal risk factors & $69(41.82)$ \\
\hline PROM>24 H & $69(41.82)$ \\
Foul smelling liquor & $20(12.12)$ \\
Maternal fever & $67(40.61)$ \\
Unclean per vaginal examination & $37(22.42)$ \\
\hline Hematological Parameters & $66(40.00)$ \\
\hline Leukocytopenia (TLC $<5000)$ & $70(42.42)$ \\
Absolute neutrophil counts < 1500 & $86(52.12)$ \\
Thrombocytopenia (platelets < 1 lakh) & $69(41.82)$ \\
RDW $\geq 18$ & \\
Elevated CRP & \\
PS (I/T Ratio >0.2, Toxic Granules, Vacuolation) & \\
\hline TLC: total leucocyte count; ANC: Absolute neutrophil count, CRP: C-reactive \\
protein, RDW: red cell distribution width, PS: peripheral smear, \\
\end{tabular}

\subsection{Microbiological Assessments}

Table 2 shows the microbiological assessments performed in neonates. Overall, $28.48 \%$ of neonates had positive sepsis screen. Abnormal CSF findings (positive cellularity on lumbar puncture CSF examination) were noted in $42.42 \%$ of neonates. Among the neonates with positive CSF cellularity, 40 (57.14\%) had positive sepsis screen. The association of abnormal CSF findings with positive sepsis screen was statistically significant $(\mathrm{p}<0.0001)$. CSF culture was performed in all neonates and was negative in all cases. Blood culture positivity was $15.85 \%$. Among the pathogens isolated on blood culture, Escherichia coli (23.07\%) and group B Streptococci (19.23\%) were common followed by the equal number of Acinetobacter spp. and Klebsiella pneumoniae ( $15.38 \%$ each), Staphylococcus aureus $(11.53 \%)$, Pseudomonas aeruginosa (7.69\%), and single case of each Coagulase-negative Staphylococcus and Enterococcus (3.84\% each).

Table 2. Microbiological assessments performed in neonates

\begin{tabular}{lc}
\hline \multicolumn{1}{c}{ Parameter } & Observations \\
\hline Positive sepsis screen & $47(28.48)$ \\
Positive CSF cellularity & $70(42.42)$ \\
Positive sepsis screen in neonates with abnormal CSF finding & $40(57.14) *$ \\
Blood culture positivity & $26(15.85)$ \\
Escherichia coli & $6(23.07)$ \\
Group B Streptococcus & $5(19.23)$ \\
Acinetobacter spp. & $4(15.38)$ \\
Klebsiella pneumoniae & $4(15.38)$ \\
Staphylococcus aureus & $3(11.53)$ \\
Pseudomonas aeruginosa & $2(7.69)$ \\
Coagulase negative Staphylococcus & $1(3.84)$ \\
Enterococcus & $1(3.84)$ \\
\hline * p value <0.0001 &
\end{tabular}




\subsection{Primary Outcome}

In our study, $136(82.43 \%)$ neonates survived whereas $29(11.57 \%)$ died (Figure 1). We observed that a significantly higher number of neonates with increased RDW died compared to that of normal RDW (27.14\% vs. $10.52 \%$, respectively; $\mathrm{p}=0.006$ ) (Table 3). Further, we observed a positive sepsis screen in a significantly higher proportion of neonates with increased RDW than normal RDW (42.85\% vs. 17.89\%, respectively; p<0.001). Similarly, a significantly higher proportion of neonates with abnormal RDW had leukocytopenia $(67.14 \%$ vs. $21.05 \%$, respectively; $\mathrm{p}<0.001)$. However, elevated CRP levels were seen in a significantly higher proportion of neonates with normal RDW ( $\mathrm{p}=0.041)$ as shown in Table 3.

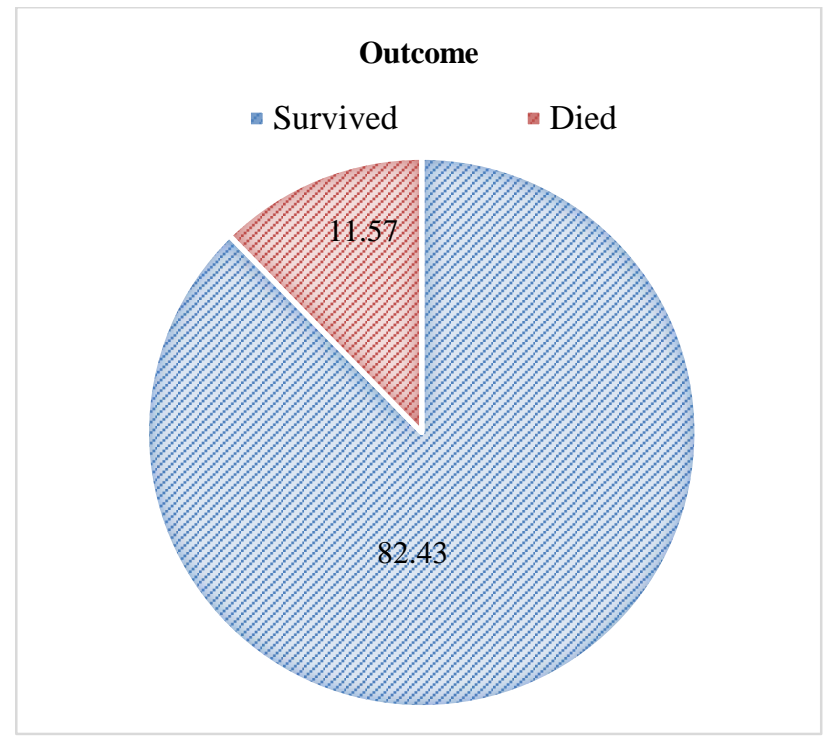

Figure 1. Outcome of neonates

Table 3. Association of abnormal RDW with sepsis parameters and death outcome

\begin{tabular}{cccc}
\hline \multirow{2}{*}{ Sepsis parameter } & \multicolumn{2}{c}{ RDW } & \multirow{2}{*}{ P-Value } \\
\cline { 2 - 3 } & Abnormal (n=70) & Normal (n=95) & \\
\hline Positive sepsis screen & $30(42.85)$ & $17(17.89)$ & $<0.001$ \\
Leukocytopenia & $47(67.14)$ & $20(21.05)$ & $<0.001$ \\
Elevated CRP levels & $30(42.85)$ & $56(58.94)$ & 0.041 \\
Death outcome & $19(27.14)$ & $10(10.52)$ & 0.006 \\
\hline
\end{tabular}

In neonates with positive blood culture, abnormal RDW was seen in $46.15 \%$ of neonates and the association was statistically significant $(\mathrm{p}<0.0001)$ as shown in Table 4 . Thus, in the diagnosis of early neonatal sepsis, RDW has a sensitivity of $46.15 \%$, specificity of $67.75 \%$, a positive predictive value of $17.14 \%$, and a negative predictive value of $85.26 \%$. Accuracy was $56.36 \%$.

Table 4. Diagnostic yield of RDW against blood culture

\begin{tabular}{cccc}
\hline \multirow{2}{*}{ RDW } & \multicolumn{2}{c}{ Blood Culture } & \multirow{2}{*}{ P-value } \\
\cline { 2 - 3 } & Positive (n=26) & Negative (n=139) & \\
\hline Abnormal $(\mathrm{n}=70)$ & $12(46.15)$ & $58(35.25)$ & $<0.001$ \\
Normal $(\mathrm{n}=95)$ & $14(53.85)$ & $81(67.75)$ & \\
\hline
\end{tabular}

\section{Discussion}

A significant observation in our study was the RDW increased in parallel with the severity of illness from sepsis to severe sepsis and septic shock. This signifies, in line with a previous study, that RDW can be a most useful predictor of mortality along with a strong indicator of sepsis severity.

In our study, RDW was found elevated in $42.42 \%$ of neonates. Martin et al. [19] also reported elevated RDW in neonatal sepsis in about $20 \%$ of enrolled subjects. The median RDW levels were significantly higher among the neonatal sepsis cases. In a study from India, Deka et al. (2020) also reported raised RDW in neonates with sepsis than 
those without sepsis. We observed a significant association of RDW with leukocytopenia. Deka et al. (2020) reported leukocytopenia in 67 subjects, of which 47 (70.14\%) had elevated RDW [14]. The association was significant (p $<0.001)$.

In our study, the association of elevated RDW with CRP was not seen as more neonates from the normal RDW group also had elevated CRP. CRP is a marker of sepsis and is therefore expected to be increased in all neonates with sepsis. However, Ellahony et al. [13] reported a strong positive correlation between RDW and CRP $(\mathrm{r}=0.8$; P $<0.0001)$. Omer and Mohammed (2020) also reported a significant association between positive blood culture, CRP and elevated RDW (p-value 0.01). Thus, RDW can be considered as a simple toll to assist the diagnosis of neonatal sepsis [20].

An increased RDW mirrors a profound dysregulation of erythrocyte homeostasis. Impaired erythropoiesis and abnormal erythrocyte metabolism and survival, that may be caused by a variety of abnormalities, such as shortening of telomere length, oxidative stress, inflammation, erythrocyte fragmentation, poor nutritional status, hypertension, dyslipidemia, and abnormality of erythropoietin function [11, 21]. All these conditions are important prognostic factors for severe morbidity and mortality. In our study, 29 (11.57\%) neonates died. Multi-centric data from India have also revealed similar $17 \%$ mortality among out born neonates [22]. In a study conducted by Jajoo et al. [23], 14.6\% of neonates died. In research done by Meshram et al. [24] 174 out of 455 neonates with clinical sepsis died, thus mortality rate being $38.24 \%$. Thus, the mortality rate in our study is comparable to other previously published studies of early-onset sepsis in neonates. Increased RDW was significantly associated with increased mortality $(p<0.006)$. Martin et al. [19] reported significantly higher median RDW levels amongst the non-survivors ( $p<0.003$ ). A study by Ellahony et al. (2017) [13] reported a positive association of RDW with mortality (OR, 1.31; 95\% CI, 1.241.39).Furthermore, Mousa and colleagues [25] identified that RDW was most sensitive (77.8\%) mortality predictor followed by mean platelet volume (MPV) $(61.1 \%)$ and platelet distribution width (PDW) (50\%). These pieces of evidence indicate the prognostic role of increased RDW in predicting mortality in neonatal sepsis.

Blood culture a definitive test to ascertain the sepsis diagnosis. Increased RDW showed a significant association with blood culture positivity. A study from Karabulut and Arcagok [26] identified that RDW and red cell distribution width to platelet ratio (RPR) was higher in suspected and proven EONS compared to control group. They further suggested that raised RPR may be used for diagnosis of EONS. With respect to the above ratio elevated RDW has a sensitivity of $44.4 \%$ and a specificity of $57.97 \%$. The sensitivity and specificity of increased RDW were $46.15 \%$ and $67.75 \%$ whereas the accuracy was $56.36 \%$. A study from Deka et al. reported that the RDW cut-off level of $17.25 \%$ had $86 \%$ sensitivity, $87 \%$ specificity, and $93.5 \%$ accuracy in diagnosing neonatal sepsis (14). Further, it is supported by the finding of a higher proportion of neonates with increased RDW having positive sepsis screen. It indicates RDW is probably suitable for establishing the early diagnosis of EONS.

Though we see encouraging results, there are certain limitations. The findings need to be confirmed in a larger sample prospectively. Also, a single reading of RDW may not be sufficient. A trend of RDW over few days of life might provide more diagnostic and prognostic insights. We did not compare the RDW in different pathogens. Also, comparison with equivalent and matched control groups may provide better estimates for the yield of RDW as a diagnostic marker in EONS.

\section{Conclusion}

In neonatal period, sepsis may be commonly observed. Early-onset neonatal sepsis (EONS) is still a leading cause of mortality and culture-positive sepsis has more risk of mortality. Sepsis screen is a proven risk predictor of EONS and mortality. Though there have been multiple markers to identify sepsis, many may not be available readily. Also, blood culture despite being a gold-standard method, is time consuming and is limited by availability. RDW is a novel and simple marker which is easily derived from complete blood count. We observed that elevated RDW ( $\geq 18)$ can be used as a marker for diagnosis of EONS as suggested by its significant association with positive sepsis screen. Furthermore, it can be a risk predictor of mortality in EONS. Current evidence also indicates that high RDW is associated with neonatal sepsis and it is an independent outcome predictor for mortality. The advantage of RDW is that cheap, easily and widely available, repetitive measures can be readily done and is not time consuming. Incorporating it in routine practice can help in early and effective management of sepsis in neonates especially in a resource-limited and peripheral settings.

\section{Declarations}

\subsection{Author Contributions}

Conceptualization, S.G., U.C. and A.M.; methodology, S.G.; software, U.C.; validation, S.G., U.C. and A.M.; formal analysis, S.G.; investigation, A.N.; resources, A.N.; data curation, A.M. and A.N.; writing-original draft preparation, S.G.; writing - review and editing, S.G.; visualization, A.N.; supervision, A.M.; project administration, A.N. All authors have read and agreed to the published version of the manuscript. 


\subsection{Funding}

The authors received no financial support for the research, authorship, and/or publication of this article.

\subsection{Ethical Approval}

All subjects gave their informed consent for inclusion before they participated in the study. The study was conducted in accordance with the Declaration of Helsinki, and the protocol was approved by the Ethics Committee of Institutional Ethics Committee (IEC), Department of Pharmacology, Govt. Medical College (GMC), Nagpur, India (112EC/Pharmac/GMC/NGP).

\subsection{Data Availability Statement}

The data presented in this study are available on request from the corresponding author.

\subsection{Conflict of Interest}

The authors declare that they have no known competing financial interests or personal relationships that could have appeared to influence the work reported in this paper.

\section{References}

[1] World Health Organization (WHO). Newborns: improving survival and well-being. 2020 September. Available online: https://www.who.int/news-room/fact-sheets/detail/newborns-reducing-mortality. (accessed on May 2021).

[2] Worldbank. UNICEF, WHO, World Bank, UN DESA Population Division. Mortality rate, under-5 (per 1,000 live births). Available online: https://data.worldbank.org/indicator/SH.DYN.MORT. (accessed on April 2021).

[3] Sankar, M. J., Neogi, S. B., Sharma, J., Chauhan, M., Srivastava, R., Prabhakar, P. K., Khera, A., Kumar, R., Zodpey, S., \& Paul, V. K. (2016). State of newborn health in India. Journal of perinatology. 36(s3), S3-S8. doi:10.1038/jp.2016.183.

[4] Basha, S., Surendran, N., \& Pichichero, M. (2014). Immune responses in neonates. Expert Review of Clinical Immunology, 10(9), 1171-1184. doi:10.1586/1744666x.2014.942288.

[5] UNICEF. Newborn and child health. Available online: https://www.unicef.org/india/what-we-do/newborn-and-child-health. (accessed on April 2021).

[6] Jehan, I., Harris, H., Salat, S., Zeb, A., Mobeen, N., Pasha, O., McClure, E. M., Moore, J., Wright, L. L., \& Goldenberg, R. L. (2009). Neonatal mortality, risk factors and causes: a prospective population-based cohort study in urban Pakistan. Bulletin of the World Health Organization, 87(2), 130-138. doi:10.2471/blt.08.050963.

[7] Oza, S., Lawn, J. E., Hogan, D. R., Mathers, C., \& Cousens, S. N. (2014). Neonatal cause-of-death estimates for the early and late neonatal periods for 194 countries: 2000-2013. Bulletin of the World Health Organization, 93(1), 19-28. doi:10.2471/blt.14.139790.

[8] Murthy, S., Godinho, M. A., Guddattu, V., Lewis, L. E. S., \& Nair, N. S. (2019). Risk factors of neonatal sepsis in India: A systematic review and meta-analysis. PLOS ONE, 14(4), e0215683. doi:10.1371/journal.pone.0215683.

[9] Zea-Vera, A., \& Ochoa, T. J. (2015). Challenges in the diagnosis and management of neonatal sepsis. Journal of Tropical Pediatrics, 61(1), 1-13. doi:10.1093/tropej/fmu079.

[10] Meem, M., Modak, J. K., Mortuza, R., Morshed, M., Islam, M. S., \&Saha, S. K. (2011). Biomarkers for diagnosis of neonatal infections: A systematic analysis of their potential as a point-of-care diagnostics. Journal of global health, 1(2), 201-209.

[11] Jo, Y. H., Kim, K., Lee, J. H., Kang, C., Kim, T., Park, H. M., Kang, K. W., Kim, J., \& Rhee, J. E. (2013). Red cell distribution width is a prognostic factor in severe sepsis and septic shock. The American journal of emergency medicine, 31(3), 545-548. doi:10.1016/j.ajem.2012.10.017.

[12] Lippi, G., Targher, G., Montagnana, M., Salvagno, G. L., Zoppini, G., \& Guidi, G. C. (2008). Relationship between red blood cell distribution width and kidney function tests in a large cohort of unselected outpatients. Scandinavian Journal of Clinical and Laboratory Investigation, 68(8), 745-748. doi:10.1080/00365510802213550.

[13] Ellahony, D. M., El-Mekkawy, M. S., \& Farag, M. M. (2017). A Study of Red Cell Distribution Width in Neonatal Sepsis. Pediatric Emergency Care, Publish Ahead of Print. doi:10.1097/pec.0000000000001319.

[14] Deka, A., \& P., A. (2020). Red cell distribution width as a diagnostic marker in neonatal sepsis. International Journal of Contemporary Pediatrics, 7(4), 820. doi:10.18203/2349-3291.ijcp20201137.

[15] Chen, J., Jin, L., \& Yang, T. (2014). Clinical study of RDW and prognosis in sepsis new borns. Biomedical Research, 25 (4), 576-579. 
[16] Garofoli, F., Ciardelli, L., Mazzucchelli, I., Borghesi, A., Angelini, M., Bollani, L., ... Stronati, M. (2013). The red cell distribution width (RDW): Value and role in preterm, IUGR (intrauterine growth restricted), full-term infants. Hematology, 19(6), 365-369. doi:10.1179/1607845413y.0000000141.

[17] Tonbul, A., Tayman, C., Catal, F., Kara, S., \& Tatli, M. M. (2011). Red cell distribution width (RDW) in the newborn: normative data. Journal of Clinical Laboratory Analysis, 25(6), 422-425. doi:10.1002/jcla.20496.

[18] Sankar, M. J., Agarwal, R., Deorari, A. K., \& Paul, V. K. (2008). Sepsis in the Newborn. The Indian Journal of Pediatrics, 75(3), 261-266. doi:10.1007/s12098-008-0056-z.

[19] Martin, S. L., Desai, S., Nanavati, R., Colah, R. B., Ghosh, K., \& Mukherjee, M. B. (2018). Red cell distribution width and its association with mortality in neonatal sepsis. The Journal of Maternal-Fetal \& Neonatal Medicine, 32(12), 1925-1930. doi:10.1080/14767058.2017.1421932.

[20] Omer, I., \& Mohammed, B. (2021). A study of red cell distribution width and neonatal sepsis at Soba University Hospital, Khartoum, Sudan. Sudanese Journal of Paediatrics, 42-47. doi:10.24911/sjp.106-1597237251.

[21] Christensen, R. D., Yaish, H. M., Henry, E., \& Bennett, S. T. (2014). Red blood cell distribution width: reference intervals for neonates. The Journal of Maternal-Fetal \& Neonatal Medicine, 28(8), 883-888. doi:10.3109/14767058.2014.938044.

[22] Mondal, G. P., Raghavan, M., Bhat, B. V., \& Srinivasan, S. (1991). Neonatal septicaemia among inborn and outborn babies in a referral hospital. The Indian Journal of Pediatrics, 58(4), 529-533. doi:10.1007/bf02750936.

[23] Jajoo, M., Kapoor, K., Garg, L., Manchanda, V., \& Mittal, S. (2015). To study the incidence and risk factors of early onset neonatal sepsis in an out born neonatal intensive care unit of India. Journal of Clinical Neonatology, 4(2), 91. doi:10.4103/2249-4847.154106.

[24] Bhongade, S., Meshram, R., \& Gajimwar, V. (2019). Predictors of mortality in outborns with neonatal sepsis: A prospective observational study. Nigerian Postgraduate Medical Journal, 26(4), 216-222. doi:10.4103/npmj.npmj_91_19.

[25] Mousa, S., Moustafa, A., \& Aly, H. (2019). Prognostic value of red cell distribution width, platelet parameters, and the hematological scoring system in neonatal sepsis. The Egyptian Journal of Haematology, 44(3), 183. doi:10.4103/ejh.ejh_12_19.

[26] Karabulut, B., \& Arcagok, B. C. (2019). New Diagnostic Possibilities for Early Onset Neonatal Sepsis: Red Cell Distribution Width to Platelet Ratio. Fetal and Pediatric Pathology, 39(4), 297-306. doi:10.1080/15513815.2019.1661051. 